\title{
MASSAGE THERAPY FOR SYMPTOM REDUCTION AND IMPROVED QUALITY \\ OF LIFE IN CHILDREN WITH CANCER IN PALLIATIVE CARE: A PILOT STUDY
}

Lara M. Genik, C. Meghan McMurtry, Shannon Marshall, Adam Rapoport, and Jennifer Stinson

Genik, Lara. McMurtry, M. Marshall, S. Rapoport, A. Stinson, J. 2020. Massage therapy for symptom reduction and improved quality of life in children with cancer in palliative care: A pilot study. Complementary Therapies in Medicine. https://doi.org/10.1016/j.ctim.2019.102263.;

Author Note

Lara M. Genik, M.A., Department of Psychology, University of Guelph, lgenik@uoguelph.ca; C. Meghan McMurtry, Ph.D., C. Psych, Department of Psychology, University of Guelph, Clinical and Health Psychologist, Pediatric Chronic Pain Program, McMaster Children's Hospital, Associate Scientist, Children's Health Research Institute, Adjunct Researcher, Department of Pediatrics, Western University, cmcmurtr@uoguelph.ca; Shannon Marshall, RMT, The Hospital for Sick Children, shannon.marshall@ sickkids.ca; Adam Rapoport, MD, The Hospital for Sick Children, Emily’s House Children’s Hospice, adam.rapoport@sickkids.ca; Jennifer Stinson, RN, Ph.D., CPNP, The Hospital for Sick Children, University of Toronto, jennifer.stinson@ sickkids.ca.

The authors are grateful to children and their families who participated in this research study as well as to the Paediatric Advanced Care Team at The Hospital for Sick Children for assistance with recruitment, and Jasmine Dunston for assistance with data collection. This project was funded by the Team for Research in Adolescent and Childhood Palliation and Grief's 
Innovation Fund. L. Genik, C.M. McMurtry, S. Marshall, A. Rapoport, and J. Stinson report that they have no competing financial interests. Word Count Including Abstract: 3366 


\begin{abstract}
Background: For children with cancer in palliative care, pain and worry are common and frequently under-managed, which negatively impacts quality of life (QOL). Massage therapy (MT) can lead to reduced pain in children with chronic illnesses. Children with cancer have experienced lower anxiety after MT. No studies have examined the effects of MT in pediatric oncology patients receiving palliative care. Objective: Conduct a MT intervention to determine intervention acceptability and initial effects on ratings of pain, worry reduction, and quality of life. Design: Pre-post single group pilot study. Setting/Subjects: Eight children with cancer (age 10-17) and one of their parents were recruited from a palliative care service.

Procedure/Measurements: Baseline (one week prior to intervention): demographics, MT expectations, QOL, and pain measures. Intervention (one month): MT was provided once per week, with children's pain and worry ratings occurring immediately before and after each MT session. Follow Up (4-6 weeks after baseline): QOL, pain, and MT/study acceptability questionnaires. Results: Participants reported significant decreases in pain following two MT sessions, and worry following one session. No significant changes in pain symptoms and QOL were found between baseline and follow up. Participants positively endorsed the study and the MT intervention, and there were no adverse effects reported. Conclusions: MT may lead to immediate decreases in pain and worry in children with cancer who are receiving palliative care, however the effects may not be sustained long term. Difficulties regarding protocol feasibility including recruitment and study compliance remain important considerations for future work.
\end{abstract} Keywords: pain, pediatric oncology, palliative care, massage therapy, quality of life, anxiety 


\section{INTRODUCTION}

Pediatric palliative care focuses on optimizing quality of life (QOL) and reducing suffering experienced by children with serious illness ${ }^{1}$. Unfortunately, symptoms experienced by dying children are frequently undermanaged through traditional interventions, resulting in increased suffering and decreased QOL. For example, although pain is reported as one of the most prevalent symptoms near end of life $e^{2,3}$, it is successfully managed less than $30 \%$ of the time $^{3}$. While effective to some degree, using pharmacological strategies on their own may be insufficient to fully treat symptoms, such as pain, experienced by these children ${ }^{3}$. Instead, a combination of pharmacological, psychological and physical management approaches may provide optimal symptom relief.

Massage therapy (MT) is a low-risk, manipulative body-based physical intervention falling under the umbrella of complementary health approaches ${ }^{4}$. Based on previous research in adult and pediatric populations with chronic illnesses, MT may help reduce symptoms such as pain and fear/anxiety and improve functioning ${ }^{1,5,6,7}$. MT may also be a promising intervention for reducing pain, fatigue and anxiety in oncology populations ${ }^{8}$. To date, a small body of literature has documented the use of MT within pediatric oncology. A systematic review of integrative clinical trials with a pediatric oncology population identified only nine studies investigating the efficacy of MT but found encouraging evidence that it may be beneficial for several symptoms including pain and anxiety ${ }^{9}$. An overview of mind-body therapies in pediatric oncology ${ }^{10}$ also highlighted several small pilot studies outlining positive psychological and physiological impacts of MT (e.g., decreased stress and anxiety). More recent use of MT in pediatric palliative care contexts has also demonstrated promising results, such as decreased symptom burden ${ }^{11}$.

The aim of this initial quantitative pre-post single group pilot study was to set the groundwork for future research on use of MT in children with cancer in palliative care. The 
specific study objectives were to: (1) determine the acceptability of the massage intervention to parents and children, and (2) obtain preliminary pre-post effectiveness data. Specifically, it was hypothesized that post MT intervention, children would report reduced pain and worry as well as improved QOL.

\section{METHODS}

\section{Recruitment and Participants}

Eight to 18-year-old children with cancer who had been referred to palliative care for either supportive or end-of-life care and one of their primary caregivers were eligible to participate. Parents and children also had to be proficient in the English language. The exclusion criteria were: (a) presence of a cognitive impairment, (b) receipt of MT within the past month, and (c) residing more than $90 \mathrm{~km}$ away from the pediatric health center. The exclusion criteria for distance from the pediatric health center was placed as participants could receive massages at home or in hospital.

Recruitment was completed at a single tertiary care pediatric institution in Canada between October 2013 and February 2017, where parents and children were initially made aware of the study by nurses on the institution's Paediatric Advanced Care Team. Data regarding the number of parents and children approached are not available. The study's massage therapist then made secondary contact with interested parents and children to provide further information and connect them with a research assistant. The final sample included eight children and a primary caregiver. See Table 1 for general demographic information about participating children and caregivers, and Table 2 for children's cancer-specific information. Several factors including low referral rate for massage therapy upon entry into the institution's palliative care service, and lack of participants meeting study inclusion/eligibility criteria (e.g., were too unwell to complete 
surveys, not proficient in English, too young, living further than 90km radius from hospital)

contributed to the study's long recruitment timeframe.

\section{Intervention}

The MT intervention consisted of four weekly massages by a registered massage therapist who had experience in palliative care settings since 2007. Massages took place either in hospital or at home as per the contract between the registered massage therapist and the hospital's palliative care service. Each massage was approximately 60 minutes in length. A balance was sought between standardization of the intervention and individualization. For example, special considerations or alterations were applied to the MT session when they were related to the child's physical care and well-being such as type of cancer, location of metastases, adverse reactions to treatments, skin fragility, edema, and open wounds or inflammation. Table 3 describes factors which remained standardized versus individualized.

\section{Procedures}

Following hospital and university ethics clearance, patients and parents meeting study criteria were approached. Parents completed informed consent with a research assistant. Children provided child-specific consent or verbal assent depending on their individual capabilities. See Figure 1 for study procedures and study timeline. Participating parents and children received reminder phone calls throughout the study related to measure completion/return of study materials and received a $\$ 20$ honorarium and certificate for participation. A research assistant completed a medical chart review which allowed the research team to gather additional medical information about the child such as number of surgeries, type of cancer, and medications prescribed.

\section{Materials}


Demographics Questionnaire. This 14-item researcher-generated, paper-based questionnaire asked parents to indicate information about themselves (e.g., age, relationship to child, ethnicity, education) and their child with cancer (e.g., age, date of cancer diagnosis, ethnicity).

Massage Related Expectations. Two analogous (parent, child) researcher-generated, paper-based questionnaires were used to determine children's massage-related experiences (e.g., Has your child ever had massage therapy before?) and expectations of the potential helpfulness of the intervention (e.g., Please rate how helpful you expect the massages to be in this study...). The parent version had 2 to 7 questions (depending on whether massage had been received in the past) whereas the child version had 2 to 5 questions.

\section{Pediatric Quality of Life Inventory 3.0 - Cancer Module (PedsQL - Cancer): Self-} Report and Parent Report Versions. The PedsQL-Cancer Module ${ }^{12}$ is a 27 -item paper-based measure of QOL which was completed by both the participating child and parent. It is composed of eight dimensions which contribute to QOL (e.g., pain and hurt, worry, procedural anxiety $)^{12}$. As per PedsQL administration protocols, a child (ages 8-12) or adolescent (ages 13-18) version of this questionnaire was used depending on participants' age. Questions on these measures are identical and as such were grouped for analyses. For each question, participants respond on a 5point Likert rating scale ranging from 0 (Never) to 4 (Almost Always) ${ }^{12}$. Individual dimension scores as well as an overall score can be calculated. Raw scores were converted to standard scores which ranged from 0 to 100 , and higher scores indicate better QOL ${ }^{12}$. In 8-18 year old children with cancer, the PedsQL 3.0 - Cancer Module has demonstrated acceptable internal consistency reliability (average $\alpha=0.72$ child, 0.87 parent report for Cancer Module Scales) ${ }^{12}$. Construct validity of the Cancer Module Scales using the known-groups method demonstrated 
differences on select self-report (nausea, treatment anxiety, worry) and parent proxy (pain, nausea, procedural anxiety, treatment anxiety, worry) subscales for children with cancer who were on treatment compared to those who had been off treatment for more than twelve months 12. Medium to large effects of intercorrelations between children's self-report and parents' proxy reports were also noted ${ }^{12}$.

PainSquad App. The Pain Squad app is a multidimensional electronic pain diary (also offered in paper-based format) designed for children between ages eight and $18^{13}$. It was used to measure children's pain (e.g., minimum, maximum, average levels, location) and pain's perceived impact on daily life (e.g., sleep, eating, medications) $)^{13}$ twice a day for four days prior to and following completion of the MT intervention. Items included in this diary assess the child's maximum, minimum, and average pain levels, locations, and durations across 12-hour periods of time ${ }^{13}$. This tool also assesses the perceived impact of the child's pain on daily life such as sleep and eating, medications, pain management strategies, and their effectiveness ${ }^{13}$. This app has been successfully used with youth (aged 8 to 18 years) diagnosed with cancer, demonstrating evidence of internal consistency $(\alpha=0.96)$ and support for construct validity, reliability, and feasibility ${ }^{13}$.

Faces Pain Scale - Revised (FPS-R). The FPS-R ${ }^{14}$ was a single item paper-based measure used to collect the child's pain ratings. The FPS-R consists of six faces representing increasing levels of pain ${ }^{14}$. To complete this measure, participants circle the face most representative of their current pain levels with scores ranging from 0 (no pain) to 10 (extreme pain $)^{14}$. The FPS-R has been primarily studied with children and adolescents over the age of five in acute, procedural and recurrent pain contexts, demonstrating strong psychometric properties including convergent validity, construct validity, and reliability ${ }^{15}$. 
Children's Fear Scale (CFS). The $\mathrm{CFS}^{16}$ is a single-item paper-based measure which was used to collect children's ratings of state worry/fear. The CFS consists of five faces showing increasing levels of fear ${ }^{16}$. To complete this measure, participants circle the face most representative of their current level of "worry" or "fear" with scores ranging from 0 (not worried at all) to 4 (the most worried or scared possible $)^{16}$. The CFS has demonstrated support for construct validity, test-retest reliability, and interrater reliability ${ }^{16}$ in the context of acute pain. It has been successfully used in settings including procedural pain ${ }^{17,18,19}$, palliative care ${ }^{20}$, perioperative $^{21}$, pediatric oncology $\mathrm{y}^{22}$ and community-based intervention settings ${ }^{23}$ with children and adolescents.

MT and Study Acceptability. This 10-item researcher-generated and paper-based questionnaire asked parents and/or participating children about the acceptability of the MT intervention as well as the study and related procedures. This questionnaire consisted of a series of ratings from 0 (Strongly Disagree) to 5 (Strongly Agree) such as 'massage therapy helped with relaxation'. It also included open-ended questions (e.g., What aspect(s) of the study did you find easiest to complete?).

\section{RESULTS}

\section{Analytic Approach and Missing Data}

Inspection of the data revealed that children's pain and worry ratings following massage interventions as well as child and parent ratings of quality of life were not normally distributed. Therefore, although we had planned parametric analyses (paired sample t-tests), we instead conducted their nonparametric equivalent, Wilcoxon signed rank tests, to account for non- 
normality ${ }^{*}$. Descriptive statistics (means, medians, standard deviations, ranges), $95 \%$ confidence intervals, and effect sizes $(r)$ were used to report outcomes.

Completion of study materials and MT intervention proved difficult to coordinate with a majority of the participants. Nevertheless, participants completed data relevant to the time points in which they were able to participate. Seven of eight participants completed baseline data. Four participants completed all MT sessions as intended by the study; two missed one massage time point, and one did not complete any massages. Five participants completed follow up data. Participants missing data on a variable needed for a specific analysis were excluded only from that particular analysis. The number of participants represented in each analysis is provided for each result. Of note, there were many challenges associated with gathering the PainSquad ${ }^{\mathrm{TM}}$ app data (e.g., missed data collection points, inability to complete the PainSquad ${ }^{\mathrm{TM}}$ app during predetermined time points) resulting in a large amount of missing data (of those who did complete the PainSquad ${ }^{\mathrm{TM}}$ there was less than $55 \%$ compliance). Therefore, these data were not analyzed.

\section{Expectations Regarding MT}

As per parent $(85.7 \% ; n=6 / 7)$ and child report $(71.4 \% ; n=5 / 7)$, the majority of children had not previously received MT. Participants anticipated the MT intervention would be helpful $(0-10 ; 10=$ Extremely Helpful $):$ parents $($ Mean $=7.00 / 10 ;$ Median $=7.00 / 10 ;$ range $=5-10 ; S D=$ $1.83 ; n=7)$ and children $($ Mean $=7.29 / 10 ;$ Median $=7.00 / 10 ;$ range $=5-10 ; S D=1.89 ; n=7)$.

\section{Preliminary Pre-Post Effectiveness of MT on Pain, Worry, and QOL}

\footnotetext{
* The overall pattern of results when comparing Wilcoxon signed rank tests to paired sample t-tests was the same [i.e., lower pain and worry following each massage; no change in QOL from baseline to follow up] but the number of analyses reaching significance differed.
} 
Wilcoxon signed rank tests revealed statistically significant decreases in self-reported pain immediately following two MT sessions, and worry immediately after one MT session (see Table 4). Data from all other time points were trending towards significant decreases in pain and worry with medium to large effects. Compared to baseline, there were no significant differences in parent or child reported overall QOL one week following completion of the MT intervention (Table 5). Similarly, there were no significant differences in parent or child reported levels of pain or worry reported on a QOL measure at one week follow up (Table 5).

\section{Acceptability of a MT Research Protocol}

Participants indicated that the study was clearly explained $($ Mean $=4.20 / 5$; Median $=$ 4.00/5; range: $3-5 ; S D=0.84$ ), and that they would encourage other families in similar situations to take part in this research $($ Mean $=4.60 / 5 ;$ Median $=5.00 / 5$; range: $4-5 ; S D=0.55)$.

Participants were neutral (i.e., did not have strong opinions) regarding the amount of materials to complete in this study $($ Mean $=3.00 / 5 ;$ Median $=3.00 / 5$; range: $2-4 ; S D=1.00)$. All participants rated the MT intervention as acceptable and agreed that it helped with muscle aches/pains (Mean $=4.80 ;$ Median $=5.00 / 5 ;$ range: $4-5 ; S D=0.45)$, lowered worry/stress $($ Mean $=4.40 ;$ Median $=$ 4.00/5; range: 4-5; $S D=0.55)$, helped with relaxation $($ Mean $=5.00 ;$ Median $=5.00 / 5$; range: 5 ; $S D=0.00)$, and improved their overall QOL $($ Mean $=4.60 ;$ Median $=5.00 / 5 ;$ range: $4-5 ; S D=$ $0.55)$.

\section{DISCUSSION}

Symptoms such as pain and worry/fear are common in children with cancer receiving palliative care, and adequate management of these symptoms is difficult ${ }^{2,3}$. Complementary therapies such as MT may show promise in further alleviating these symptoms while also improving QOL ${ }^{5,6}$. MT is low-risk, currently being used in some pediatric oncology services, and 
has demonstrated positive effects in both adults and children with chronic illnesses ${ }^{4,5,6}$. To date, research has not directly evaluated the use of this approach for children with cancer receiving palliative care. Despite the challenges accompanying research in palliative populations, this work is important to determine factors such as the impact of MT, frequency, and dosage. The purpose of this study was to obtain preliminary acceptability and pre-post effectiveness data associated with MT's impact on pain, worry, and overall QOL.

Participants reported positive massage-related expectations and found the study and intervention to be acceptable. Further, participants reported significant decreases in pain and worry following select time points. Results were trending towards significant decreases at all time points with medium to large effect sizes. These findings are similar to those reported in other massage therapy related research with other populations ${ }^{5,6}$. Importantly, it is possible that external factors may have affected the impact of the MT intervention at each time point. For example, as children approached end of life, it is possible that their illness-related pain symptoms may have become difficult to relieve. Similarly, symptoms of worry may fluctuate and be harder to alleviate at times of greater stress. Nevertheless, these results suggest potential for a MT protocol as employed in this study to have positive immediate effects on children's symptoms near end of life. MT did not translate into longer lasting improvements as measured by the PedQL from baseline to one week following completion of the MT intervention; yet, perhaps it is noteworthy that there was no change in children's QOL over the 6 week course of the study when one might expect it to decrease given the child's proximity to end of life.

Despite an attempt to create a feasible study protocol given the target sample, several challenges occurred during the study, many of which are consistent with previous research in palliative care settings (e.g., recruitment and retention, compliance difficulties) ${ }^{24}$. As described 
earlier in the manuscript, recruitment was slow and challenging. Similarly, aspects of the study design proved difficult and related in many ways to the unpredictable and declining nature of participants' health (e.g., another more critical appointment interfering with scheduled intervention, feeling too unwell to complete questionnaires). These findings bring to light important questions about how to develop feasible research projects directed towards vulnerable groups of people. Use of participatory action research (e.g., involving stakeholders such as parents $)^{25}$ in developing similar study protocols would likely be helpful.

\section{Strengths, Limitations, and Future Directions}

To our knowledge, this was the first study to directly evaluate a MT intervention for children with cancer in palliative care. Where possible, well-developed, psychometrically sound research questionnaires and tools (e.g., PedsQL, FPS-R) were used to maximize validity and reliability and inform future research. However, this was also balanced with the need for standardization and individual tailoring of the MT intervention itself, allowing youth to communicate the focus of the treatments and treatment locations. This pilot allowed researchers to explore the use of MT within the context of a research study in a population that poses a number of challenges to research.

There are also study limitations to consider. First, the pilot study did not include a control group. Second, recruitment was slow and challenging; consideration of research that involves multiple sites may help improve recruitment. Third, despite a number of strategies to reduce participant burden and assist with protocol compliance (e.g., reminder phone calls, options to complete MT intervention in hospital or at home, mailing study materials, and provision of an honorarium for study participation), this remained problematic and further limits the validity of the results. Although the difficulty with protocol compliance is unsurprising given the challenges 
associated with research in palliative care ${ }^{24,26}$, those planning future studies will want to further consider the demand that a study protocol may have on participants and ways to provide support in this regard. For example, in a palliative care context, one may need to spend extra time sitting with participants to help them complete questionnaires, embed them into other paperwork they are already completing for other purposes, or limit the number of questionnaires even further than was done in this pilot study.

Future work should continue to evaluate the feasibility of studying MT in this population, the impact of MT on pain and worry in pediatric oncology patients near end of life, as well as the qualitative impact of MT on children. Better understanding patient needs and the impact that MT can have on their cancer-related symptoms and overall well-being could be helpful for children with cancer in both inpatient and outpatient settings alike. This may also include learning more about the impact of MT interventions implemented throughout the course of one's journey with cancer versus only at the palliative care/end of life stage.

\section{CONCLUSIONS}

Preliminary results from this study are encouraging for the use of MT as an intervention to decrease immediate pain and worry. There are a number of challenges associated with research in this population, and careful consideration to the methodology and design of related research is critical. Future work in this area could be beneficial in providing further evidence of the benefits MT may provide in improving a child's quality of life. 


\section{References}

1. Goldman A, Hain R, Liben S (Eds.): Oxford textbook of palliative care for children. Oxford University Press 2012.

2. Goldman A, Hewitt M, Collins GS, et al. Symptoms in children/young people with progressive malignant disease: United Kingdom Children's Cancer Group/Paediatric Oncology Nurses Forum survey. Ped 2006;117:e1179-e1186.

3. Wolfe J, Grier HE, Klar N, et al. Symptoms and suffering at the end of life in children with cancer. N Engl J Med. 2000;342:326-333.

4. National Center for Complementary and Integrative Health (NCCIH): What is complementary and alternative medicine? Bethesda, MD: National Institutes of Health, 2011.

5. Field T, Diego M, Hernandez-Reif M: Massage therapy research. Dev Rev 2007;27:75-89.

6. Moyer CA, Rounds J, Hannum JW. A meta-analysis of massage therapy research. Psychol Bull. 2004;130:3-18.

7. Field T: Massage therapy research review. Comp Ther Clin Prac 2016; 24:19-31.

8. Boyd C, Crawford C, Paat C.F, et al. The impact of massage therapy on function in pain populations - A systematic review and meta-analysis of randomized controlled trials: Part II, cancer pain populations. Pain Med. 2016;17;1553-1568.

9. Radossi AL, Taromina K, Marjerrison S, et al. A systematic review of integrative clinical trials for supportive care in pediatric oncology: a report from the International Society of Pediatric Oncology, T\&CM collaborative. Supp Care Cancer. 2018;26;375-391.

10. Kanitz JL, Camus MEM, Seifert G: Keeping the balance-an overview of mind-body therapies in pediatric oncology. Comp Ther Med. 2013;21;S20-S25.

11. Weekly T, Riley B, Wichman C, et al. Impact of a massage therapy intervention for pediatric palliative care patients and their family caregivers. J Pall Care. 2018. doi: $10.1177 / 0825859718810727$

12. Varni JW, Burwinkle TM, Katz ER, et al. The PedsQL ${ }^{\mathrm{TM}}$ in pediatric cancer: reliability and validity of the pediatric quality of life inventory ${ }^{\mathrm{TM}}$ generic core scales, multidimensional fatigue scale, and cancer module. Cancer. 2002;94(7), 2090-2106.

13. Stinson JN, Jibb LA, Nguyen C, et al. Construct validity and reliability of a real-time multidimensional smartphone app to assess pain in children and adolescents with cancer. Pain. 2015;156(12), 2607-2615. 
14. Hicks CL, von Baeyer CL, Spafford PA, et al. The Faces Pain Scale-Revised: toward a common metric in pediatric pain measurement. Pain. 2001;93(2);173-183.

15. Tomlinson D, von Baeyer CL, Stinson JN, et al. A systematic review of faces scales for the self-report of pain intensity in children. Am Acad Peds. 2010; 126(5); e1-e31.

16. McMurtry CM, Noel M, Chambers CT, et al. Children's fear during procedural pain: preliminary investigation of the Children's Fear Scale. Health Psych. 2011;30(6), 780788.

17. Şahiner NC, Inal S, Akbay AS. The effect of combined stimulation of external cold and vibration during immunization on pain and anxiety levels in children. $J$ Perianes Nurs. 2015;30(3), 228-35.

18. Stoltz P, Manworren RC. Comparison of children's venipuncture fear and pain: Randomized controlled trial of EMLA® and J-Tip Needleless Injection System®. J Ped Nurs. 2017;37, 91-96.

19. Hyland EJ, D’Cruz R, Harvey JG, et al. An assessment of early child life therapy pain and anxiety management: A prospective randomised controlled trial. Burns. 2015;41(8), 1642-1652.

20. Thrane SE, Maurer SH, Ren D, et al. Reiki therapy for symptom management in children receiving palliative care: a pilot study. Am J Hosp Palliat Med. 2017;34(4), 373-379.

21. Hackmann T, Haworth RA, Hong P, et al. Accuracy of children's perioperative memories. AORN J. 2017;105(6), 605-612.

22. Jibb LA, Birnie KA, Nathan PC, et al. Using the MEDiPORT humanoid robot to reduce procedural pain and distress in children with cancer: A pilot randomized controlled trial. Ped Blood Cancer. 2018;65(9), e27242.

23. Dalley JS, McMurtry CM. Teddy and I get a check-up: A pilot educational intervention teaching children coping strategies for managing procedure-related pain and fear. Pain Res Manag. 2016;2016, 1-12.

24. Kutner J, Smith M, Mellis K, et al. Methodological challenges in conducting a multi-site randomized clinical trial of massage therapy in hospice. J Palliat Med. 2010;13:739-744.

25. Jagosh J, Macaulay AC, Pluye P, et al. Uncovering the benefits of participatory research: implications of a realist review for health research and practice. Milbank Quarterly. 2010; 90(2), 311-346.

26. Rapoport A. Addressing ethical concerns regarding pediatric palliative care research. Arch Ped Adol Med. 2009;163(8), 688-691. 